\title{
Experimentelle histologische Untersuchung uber die Struktur der Lungenalveolen des \\ Kaninchens, insbesondere über die Auskleidungsepithelzellen der Alveolenwand.
}

\author{
Von \\ Kiyoharu Tamaru \\ Anatomisches Institut, Keio-Gijuku Universität, Tokyo. \\ (Direktor: Prof. Dr. T. Taniguchi)
}

\section{Einleitung.}

Die Frage über die Zusammensetzung der Auskleidungsepithelien und die Struktur der Alveolenwand bei höheren Wirbeltieren ist bis heute von vielen Autoren umstritten und noch nicht abgeklärt. Eberth(1862), Elenz (1864), Kölliker (1881) u.a. behaupteten das Vorkommen des sog. respiratorischen Epithels, nämlich die Alveolenwand ist von den kernlosen Platten und den kernhaltigen kubischen Zellen ausgekleidet, die ersteren bekleiden die dem Lumen der Alveolen zugekehrten Flächen des Blutkapillarennetzes. Diese Meinung stützen Ogawa (1920), Bensley (1935), Macklin (1936), Miller ('32, '47) u. a. Dagegen zweifelt Oppel ('05) das Vorkommen der kernlosen Platte an, das wurde dann von Policard ('26), Seeman ('31), Akutsu ('33), Clara ('36), Akazaki und Hozaka ('42), Baba ('48), Shimai und Tamaru ('51), Loosli ('35, '51) und Kammel ('52) verneint.

Seeman, Clara, Shimai und Tamaru, Loosli behaupten, da $B$ die kernhaltigen, kubischen Zellen an der Alveolenwand diskontinuierlich angeordnet sind und daß die dem Alveolenlumen zugewandte Fläche der in Alveolenwand reichlich vorhandenen Blutkapillaren zum Teil keine epitheliale Auskleidung besitzt, so da $B$ sie nur von einer dünnen Faserschicht bekleidet in dem Alveolenlumen bloßgelegt ist. Nach Dogliotti-Amprino ('32), Bunsinco ('33), Bremer ('35), Peterson ('35) und Hayek ('51) ist die betreffende Fläche der Blutkapillaren teils von dem flügelartigen Protoplasmafortsatz der kernhaltigen kubi- 
schen Zellen beständig oder unter bestimmten physiologischen Zuständen bedeckt.

Kürzlich verneint Frank Low ('52) auf Grund seiner Beobachtung mittels des Elektronenmikroskops das Vorkommen der kernlosen Platten und sagt, da $\beta$ das Alveolenepithel die Alveolenwand kontinuierlich auskleidet. Ich habe nun auf Grund unserer früheren Untersuchung ('51) eine experimentelle Untersuchung durchgeführt, um die obenerwähnte Frage des respiratorischen Epithels, besonders die topographische Beziehung zwischen den Blutkapillaren und dem Epithel weiterhin auszuklären. Es gibt eine Reihe von Berichten über die histopathologische Untersuchung der experimentellen oder klinischen Kollapslungen, doch wurde die histologische oder zytologische Untersuchung des Alveolenepithels beim künstlichen Pneumothorax nur von Loosli ('32), Hayek ('51) und Kurita ('51) vorgenommen. Ich habe an der Lunge von gesunden Kaninchen den experimentellen Pneumothorax über 3 Monate lang durchgeführt, das Alveolenlumen zusammenziehen lassen, und die dementsprechenden Veränderungen der Auskleidungsepithelien und der Blutkapillaren der Alveolenwand histologisch geforscht. Im Folgenden möchte ich über die Befunde berichten.

\section{Material und Methode.}

Die Versuchstiere waren 30 gesunde Kaninchen mit $2-2.5 \mathrm{Kg}$ Körpergewicht, von denen 10 zur Kontrolle dienten. Der künstliche Pneumothorax wurde mittels des Terao'schen Apparats so erzeugt, indem in die rechte Pleurahöhle anfangs $20 \mathrm{cc}-30 \mathrm{cc}$, später jeden 3.-7. Tag $50 \mathrm{cc}-100 \mathrm{cc}$ Luft zugeführt wurde. Die längste Behandlungsdauer war 3 Monate. Von den durch Luftembolie abgetöteten Tieren wurden sofort 3-5 $\mathrm{mm}^{3}$ Stückchen der Lungen herausgeschnitten, welche mit $70 \%$ Alkohol, 10\% Formol, Zenker-Formol, Levi' und Champy'schem Gemisch fixiert wurden. Dabei sinken die hinreichend kollabierten Lungenstückchen in der Fixierungsflüssigkeit, aber bei reichlichem Luftgehalt in den Alveolen schwimmen die Lungenstückchen in der Flüssigkeit, so wurden die Stückchen in geschlossenen Fixierungsfläschchen, in denen die Luft mit der Wasserstrahlpumpe abgezogen wurde, fixiert, um die Restlüfte in Alveolen zu entfernen und ein vollständiges Eindringen der Fixierungsflüssigkeit in die Stückchen zu erzielen. Es wurden 3-5 $\mu$ Paraffinschnitte hergestellt. Die Färbung geschah hauptsächlich mit Hämatoxylin (Hansen)-Eosin, Eisen-Hämatoxylin nach Heidenhain und Azanfärbung, für die elastischen Fasern mit Elastin- 
färbung nach Weigert, für die Gitterfasern mit Brom-Silber Methode und Bielschowsky-Maresch'scher Methode, für die kollagenen Fasern mit van Gieson'scher Färbung, für den feineren Bau der Zellen mit Kull'scher Anilinfuchsin-Aurantiafärbung. Die Azanfärbung, van Gieson' und Weigert'sche Färbung gaben bei den mit Überjodsäure vorbehandelten Paraffinschnitten je eine gute Faserfärbung.

\section{Eigene Befunde.}

1. Über die Alveolenwand des normalen Kaninchens.

Die Entwicklung des interstitiellen Gewebes der Alveolenwand ist beim normalen Kaninchen relativ gut. An der Alveolenwand sind die kubischen Zellen unregelmäßig angeordnet, indem sie einzeln oder zu kleineren Gruppen liegen. Diese Zellen sind sog. Alveolenepithelien, deren Grenze bei Farbung mit Hämatoxylin-Eosin, Eisen-Hämatoxylin oder Kull'scher Methode sehr deutlich beobachtet wird. Die Zellen zeigen entsprechend dem Ausdehnungszustande der Alveolenwand ziemlich verschiedene Formen, nämlich im allgemeinen kubische, mitunter auch kugelige oder flache. Das Zytoplasma erscheint bei Färbung mit Hämatoxylin-Eosin und Azanfärbung relativ hell, und unter den Zellen gibt es solche, welche in dem Zytoplasma netzförmige retikuläre Struktur oder granulierte Masse aufweisen. Der Kern ist gegenüber der Größe des Zelleibes sehr groß, im allgemeinen lumenwärts gelegen und besitzt eine mit Hämatoxylin ziemlich gut färbende Kernmembran; auch kann man 1-2 Kernkörperchen deutlich bemerken. Die Chromatingranula sind im Karyoplasma nahezu gleichmäßig verteilt. Wie oben erwähnt, ist die Anordnung der Epithelzellen unregelmäßig und diskontinuierlich, so daß man in den Stellen, wo die Alveolenwand der Epithelien entbehrt, den Eindruck gewinnt, als ob die Kapillaren im Alveolenlumen entblößt seien. Es ist besonders ein bemerkenswerter Befund, daß die der Kapillarenwand anliegende kubische Epithelzelle nach der im Alveolenlumen bloßliegende Fläche der betreffenden Kapillare einen flügelartigen Protoplasmafortsatz entsendet. Bei der mit Eisen-Hämatoxylin gefärbten oder nach Kull'scher Methode behandelten Präparaten erscheinen die stäbchenförmigen und granulären, mitunter auch perlschnüartigen Mitochondrien in Epithelzellen, welche im ganzen Zytoplasma nahezugleichmäßig verbreitet sind. Auch finden sie sich im genannten Protoplasmafortsatz. Man kann somit ersehen, daß dieser Protoplasmafortsatz einen Teil der Epithelzellen darstellen soll. Nach meiner Beobachtung ist der Protoplasmafortsatz der Epithelzellen aber 
nicht genug lang gestreckt, um die bloßliegende Kapillarenwand vollkommen zu bedecken. Die Blutkapillaren der Alveolenwand sind stark entwickelt und zeigen einen stark geschlängelten Verlauf.

Die elastischen Fasern der Alveolenwand treten bei Weigert'scher Färbung außerordentlich reichlich in Erscheinung. Im dünnen Schnittpräparat sehen die Fasern nicht kontinuierlich aus, verlaufen nach verschiedene Richtungen innerhalb der Alveolenwand. Die dicken elastischen Fasern verlaufen im allgemeinen das Zentrum der Alveolenwand, während die von ihnen verästelten feineren Fasern in das interstitielle Gewebe der Alveolensepten sowie der Alveolenwand hineindringen und sich zwischen den Epithelzellen und den Blutkapillaren finden. Die abermals verästelten wellenförmigen feinesten Fasern umgeben unmittelbar die Epithelzellen und die Blutkapillaren. Bei der normalen Lunge ist der wellenförmige Verlauf der elastischen Fasern im allgemeinen nicht so auffallend. Bei den mit Überjodsäure vorbehandelten mit Weigert'scher Elastinfärbung gefärbten Präparaten sind die sehr feinen Fasern deutlich gefärbt, und auf der im Alveolenlumen bloßliegenden Kapillarenfläche werden die feinen elastischen Fasern aufgefunden. Bei Azanfärbung erscheinen in der Alveolenwand verschieden dicke Fasern in reichlicher Menge, bei van Gieson'scher Färbung sind die relativ feine Fasern im interstitiellen Gewebe netzartig vorhanden. Am Alveoleneingang, Anfangsteil der Alveolengänge oder der Alveolenwand befinden sich mitunter Abschnitte, in welchen gegenüber der Menge des interstitiellen Gewebes dicke Fasern reichlich vorkommen. Auch an der im Alveolenlumen bloßliegenden Oberfläche der Blutkapillaren finden sich die durch Azanfärbung und van Gieson'sche Färbung angefärbten feinen Fasern. Bei Beobachtung der Versilberungspräparate gewinnt man die Befunde, da $B$ die feinen Gitterfasern, welche von den im interstitiellen Gewebe der Alveolensepta verlaufenden dicken Fasern abzweigen, die Blutkapillaren und die Alveolenepithelzellen umgeben und ein Netzwerk bilden. Die Gitterfasern zwischen den Blutkapillaren und den benachbarten Alveolenepithelzellen sind relativ dick und deutlich ausgeprägt. Die Blutkapillaren sind durch das Netzwerk der feinen Gitterfasern umgeschlungen.

2. Über die Kaninchenlunge bei dem künstlichen Pneumothorax.

Bei kurzdauernden künstlichen Pneumothorax schrumpft teilweise die Lunge, besonders ist im allgemeinen im Oberlappen die Zusammenziehung bemerkenswert. Sonst ist im Vergleich mit der normalen Lunge anscheinend kein Unterschied $\mathrm{zu}$ erkennen. Die durch lang- 
dauernden künstlichen Pneumothorax hinreichend zusammengezogene Lunge sieht von der normalen ganz vcrschieden aus, ihre Oberfläche ist glatt, auch der Farbenton dunkel rötlich und zeigt das Aussehen der parenchymatösen Organe wie Niere, Leber und Milz. An der Pleura ist die Verwachsung nicht $z \mathrm{u}$ beobachten.

\section{Allgemeine Befunde.}

Die hinreichend kollabierte Lunge zeigt auf den ersten Blick das Bild von Lungengewebe der embryonalen Zeit, auch finden sich Abschnitte, in welchen das Alveolenlumen beinahe kaum zu unterscheiden ist. An den Arterien, Venen und Blutkapillaren der Alveolen wird durchweg Blutstauung bemerkt, das Gefäßlumen, besonders das Querschnittsbild der Kapillaren ist größtenteils rundlich und deutlich ausgebreitet. Das Bronchiolenlumen ist gedrückt, aber das Lumen bleibt erhalten, in welchem sich einige sekretähnliche Masse oder freie Zellen befinden. Unter solchen Zellen gibt es solche, die in den Lücken der Bronchiolenepithelien eingeschaltet sind. Diese Zellen sind gewöhnlich größer als die Alveolenepithelzellen, auch ist ihr Kern relativ groß. Eine Anzahl dieser Zellen besitzen im Zytoplasma auch granulierte Substanz, und ähneln den im Alveolenlumen reichlich vorkommenden sog. Staubzellen. Die Karyolyse oder Karyorrhexis und Anschwellung des Zelleibes sind nicht zu bemerken. Die Epithelzellen des Bronchiolus sind im allgemeinen regelmäßig angeordnet, der Kern erscheint bei Hämatoxylin-Eosinfärbung gegenüber den Zellen des Alveolenepithels sehr markant. Die Tela submucosa ist im allgemeinen dün, doch lassen die Knorpelstücke und die glatte Muskulatur gegenüber der normalen Lunge keine Differenz konstatieren. Die elastischen Fasern sind im allgemeinen dick, aber ihre Windung ist mehr sanft. Die Wände der mittelgroßen Venen sind gegenüber der normalen Lunge verdickt, auch sind die Muskelfasern der Arterienwand reichlich vorhanden. Die elastischen Fasern der Gefäßwand sind ziemlich dick, der wellenförmige Verlauf ist ausgeprägt, dies ist besonders der Fall in der Arterienwand einschließlich der kleinen Arterien. Die Gefäße der Pleura zeigen im Vergleich mit den der normalen Pleura keine nennenswerte Verdickung. Beim 2-3 Monate lang andauernden Pneumothorax nimmt im allgemeinen die Hyperämie der Arterien und Venen sowie Alveolenwandkapillaren ab. Die Arterien- und Venenwände sind verdickt, dort wird die Anordnung der kollagenen und Gitterfasern gegenüber der normalen Lunge dichter, auch ist die Menge der elastischen Fasern auffallend zugenommen. Beim dreimonatig andauernden Pneumothorax 
nimmt im Bronchiolen- und Alveolenlumen die sekretähnliche Substanz $z \mathbf{u}$, dagegen ist in der Umgebung des Bronchiolus oder im interstitiellen Gewebe der Alveolenwand die Vermehrung der Zellen oder die Zunahme der histiozytären Zellen nicht $z \mathrm{u}$ beobachten. Die Anordnung der Epithelzellen des Bronchiolus wird unregelmäßig, desgleichen auch die Verlaufsrichtung der Muskelfasern der Bronchiolenwand. Die deutlich wellenförmig gewundenen elastischen Fasern nehmen auch an Dicke $z \mathrm{u}$, auch wird die Anordnung der Gitter- und kollagenen Fasern dichter und ihre Menge reichlicher. Die Faserstruktur der Pleura ist auffallend dicht und ihre Dicke nimmt $z u$, besonders ist die Vermehrung der kollagenen Fasern zu bemerken.

\section{II. Über die Alveolenwand.}

Der Schrumpfungsgrad der Alveolen ist unbestimmt, denn auch bei kurzandauernder Behandlung finden sich hinreichend zusammengezogene Teile. Trotz langdauernder Pneumothoraxbehandlung finden sich auch solche, wo das Alveolenlumen ziemlich luftreich ist. Im allgemeinen ist die Schrumpfung deutlicher im Zentralteil der Lungenlappen als in der Subpleuralgegend. Es gibt aber solche Fälle, bei denen die Schrumpfung umgekehrt in der dicht unterhalb der visceralen Pleura befindlichen Zone deutlich ist.

Beobachtet man die Abschnitte, wo die Schrumpfung der Alveolen vollkommen erzielt worden ist, grenzen die Alveolenwände aneinander, die Alveolenlumen findet sich im Lungengewebe als einfache Spalten vor, die Alveolenwand ist verdickt. Bezüglich der Blutkapillaren der Alveolenwand vermindern sich die in dem Alveolenlumen bloßliegenden Teile im Vergleich mit der normalen Lunge, so da $B$ die Blutkapillaren auf dem ersten Blick aussehen, als ob sie innerhalb des Parenchyms vorhanden seien. Wenn man aber solche Bilder eingehend beobachtet, so ersieht man, daß die Kapillaren infolge dem Zusammenziehen des Alveolenlumens von den Epithelzellen der Umgebung umgeben werden, ohne da $B$ aber die Epithelzellen, welche die entblößte Blutkapillarenoberfläche bedecken, wirklich vorhanden sind. Bei den Alveolen von leichtem Schrumpfungszustand schlingen die Blutkapillaren und treten im Alveolenlumen etwas aufgetrieben hervor. Bei einem Fall von einmonatlicher Pneumothoraxbehandlung sind die Blutkapillaren hyperämisch, bei Hämatoxylin-Eosinfärbung sind viele eosinophile Zellen zu sehen, auch kommen in dem Zytoplasma des Alveolenepithels die eosinophile Granula vor. Die Blutkapillarenwand, welche in Alveolenlumen bloßliegt, ist viel dünner als bei der normalen Lunge. 
Die Alveolenepithelzelle zeigt im allgemeinen die Neigung, sich abzurunden, so daß das Bild der Absendung der Protoplasmafortsätze wie bei der normalen Lunge kaum aufzufinden ist. Auch gibt es deutlich vakuolisierte Zellen. Bei den abgerundeten Zellen scheinen die Kerne und die Kernkörperchen an Größe zuzunehmen. Die Karyoplasma wird heller und das Chromatin verteilt sich im allgemeinen im Randteil des Kerns dichter. Bei den Präparaten, bei denen die Schrumpfung der Alveolen schwach ist, wölben die Blutkapillaren der Alveolenwand in das Alveolenlumen vor, die Alveolenwand ist geschlängert und zeigt deutlich Unebenheit; die Abrundung der Epithelzellen ist leichtgradig, ihre Kerne sind mehr hell und die Chromatingranula verteilen sich wie bei der normalen Lunge meistenfalls zerstreut im Kernraum. Die Staubzellen, die in der normalen Lunge entlang der Alveolenwand oder in dem Alveolenlumen frei vorhanden sind, zeigen in der kollabierten Lunge mannigfaltige Gestalten und erscheinen, als ob sie die entblößte Blutkapillarenoberfläche oder ihr anliegen. Auch in diesem Falle sind an der Berührungsfläche der Blutkapillarenwand mit den Staubzellen weder die Vermehrung der Fasern noch die Verdickung der Kapillarenwand zu beobachten.

\section{III. Über das Fasersystem der Alveolenwand.}

Bei Azanfärbung treten blau gefärbte Fasern in reichlicher Menge hervor, besonders sind die die Blutkapillaren umgebenden Fasern auch an der in das Alveolenlumen entblößten Blutkapillarenfläche als ziemlich dicke Fasern deutlich zu bemerken. Bei dem langdauernden künstlichen Pneumothorax sind diese Fasern an der oberflächlichen Schicht der Alveolenwand gut entwickelt. Die im interstitiellen Gewebe der Alveolenwand verlaufenden elastischen Fasern nehmen in den meisten Fällen an Dicke zu; auch die entlang der Alveolenwand verlaufenden, die Alveolen zirkulär umgebenden Fasern und die an der entblößten Blutkapillarenfläche laufenden treten gegenüber der normalen Lunge sehr deutlich hervor. Beim einmonatlichen künstlichen Pneumothorax zeigen die Fasern an der entblößten Blutkapillarenfläche gegenüber der normalen Lunge keine bemerkenswerte Unterschiede. Bei der Betrachtung der mit Brom-Silber und nach Bielschowsky-Maresch' scher Methode behandelten Präparaten erscheinen in der Mitte des interstitiellen Gewebes der Alveolenwand ziemlich dicke Gitterfasern, während an den Fasern der entblößten Blutkapillarenfläche nennenswerte Änderungen nicht $z u$ beobachten sind. Bei den Versuchsfällen 
ist das Netzwerk der Gitterfasern in den Alveolensepta deutlicher und komplizierter als bei der normalen Lunge.

3. Über die Kontrollunge der entgegengesetzten Seite.

Beim Betrachten der linken Lunge der Versuchskaninchen, bei denen in der rechten Lunge der künstliche Pneumothorax durchgeführt worden war, ist das Alveolenlumen im allgemeinen erweitert und die Schlingelung der Blutkapillaren in Alveolenwand deutlich. Man kann aber gewiße Fälle finden, bei denen das Alveolenlumen sehr deutlich erweitert, dementsprechend die Alveolenwand ausgedehnt ist und die darin vorhandenen Blutkapillaren auch verlängert sind. Das Alveolenepithel ist diskontinuierlich, auch ist der Zelleib im allgemeinen länglich gestreckt und platt, doch sind die Bilder, daß die Expansion des Zytoplasmafortsatzes der Epithelzellen die entblößten Blutkapillarenoberfläche überdeckt, vermißt.

In dem Teil, wo die Alveolenwand deutlich ausgedehnt ist, scheint die Zahl der Epithelzellen auffallend vermindert zu sein, und die Alveolenwand gibt den Eindruck, als ob sie nur aus dem interstitiellen Gewebe bestehe. Am Knotenpunkt (nach Seemann) der ausgedehnten Alveolensepta sind die Bindegewebsfasern des interstitiellen Gewebes gut entwickelt. Das Zytoplasma der Alveolenepithelzellen zeigt im allgemeinen netzartige Struktur, die Kernmembran ist dünn, die Färbbarkeit des Karyoplasma schwach, und die Struktur des Kerns ist etwas undeutlich; häufig ist die Verteilung der Chromatingranula zerstreut.

Die sog. Staubzellen finden sich innerhalb des Alveolenlumens oder der Alveolenwand anliegend, doch sind dieselben in der letzteren Lage von denen bei Pneumothorax insofern anders, als sie oft rundliche Formen zeigen. Auch finden sich in der ausgedehnten Alveolenwand ziemlich dicke elastische Fasern, davon zweigen die feinen $\ddot{A}$ ste, welche weiter die Epithelzellen und Blutkapillaren umgeben; ihre wellenförmige Windung ist langsam, indem sie mitunter sogar kaum zu bemerken ist. Die zur Alveolenwand ringförmig verlaufenden elastischen Fasern sind besonders deutlich ausgeprägt. Die Gitterfasern sind in viel reichlicher Menge als die elastischen Fasern vorhanden, doch zeigen die Menge und Struktur der Gitterfasern gegenüber der normalen Lunge keine Veränderung. Sie umgeben netzartig die Epithelzellen und die Blutkapillaren. 


\section{Zusammenfassende Betrachtung.}

Über die Auskleidungsepithelzellen der Alveolenwand.

Die nur auf dem Versilberungsbild der Lungenalveolen begründete Auffassung, da $B$ das Alveolenepithel aus zwei Arten Epithelzellen, den kernlosen Platten und den kernhaltigen kubischen Zellen, bestehen soll, ist heute schon etwas antik geworden. Die von verschiedenen Autoren angegebenen Versilberungsbilder sind für den Beweis des Vorhandenseins der kernlosen Platte nicht hinreichend, auch konnten die embryologischen, experimentell-histologischen und pathohistologischen Untersuchungen das Vorkommen der kernlosen Platte nicht feststellen. Heute neigen die Forscher die Ansicht zu unterstützen, daß in höheren Wirbeltieren die kleinen kernhaltigen kubischen Epithelzellen entgegen der eigentlichen Natur des Epithels eine diskontinuierliche Anordnung zeigen und in den Maschenräumen der Blutkapillarennetze der Alveolenwand vorhanden sind. Über die Struktur der Alveolenwand gibt es heute viele Meinungsverschiedenheiten, besonders die Frage über das Verhalten der Blutkapillaren gegenüber Alveolenlumen in der Stelle, wo die Alveolenepithel unterbrochen ist, ist noch offen. Die Histogenese des Alveolenepithels ist auch noch nicht endgültig aufgeklärt.

Ich möchte auf Grund unserer eigenen Befunde an der normalen Kaninchenlunge und der Kaninchenlunge bei den Erzeugung des künstlichen Pneumothorax und unter Berücksichtung der Literaturen die obenerwähnten verschiedenen Fragen diskutieren.

Bezüglich der in der Alveolenwand vorkommenden kernhaltigen kubischen Zellen wird von vielen Autoren angeführt, daß diese Zellen sich durch gewisse Reize vermehren, deformieren und die phagozytenartige Funktion und Gestalt gewinnen. Jedoch behaupten eine Anzahl Autoren auf Grund der Ergebnisse, da $B$ das Alveolenepithel der embryo. nalen Zeit in das postnatale diskontinuierliche Alveolenepithel unmittelbar fortsetzt, die epitheliale Natur der kubischen Epithelzellen, während andere Autoren der Meinung sind, daß die betreffenden Zellen von mesenchymaler Natur sind, da diese Zellen unter Umständen sich vermehren und in Makrophagen mit phagozytierender Tätigkeit umwandeln können.

Clara ('36) benannte diese spezialisierte Epithelzellen Epicyten, Busino ('33) Pneumocyten. Nach Lang ('25) stammen diese Zellen von dem Bindegewebe ab, so benannte er sie Septalzellen. Außerdem führte Bargmann ('35) diese Zellen auf Pericyten, Fried ('34) auf Histiocyten, und Robertson ('33) auf Makrophagen zurück. An un- 
serem Institut wiesen früher Baba ('48) und Shimai ('51) in ihren histogenetischen Untersuchungen auf die epitheliale Natur dieser Zellen hin. In meinem künstlichen Pneumothoraxversuch ist es schwierig, die Histogenese des Alveolenepithels zu verfolgen. Wenigstens war es unmöglich, auf Grund der Ergebnisse der Versuche die Wucherung des Alveolenepithels $z \mathbf{u}$ erörtern. Obwohl das Vorkommen der kleinen kernhaltigen kubischen Epithelzellen in der normalen Lunge wie auch in der Lunge bei dem künstlichen Pneumothorax mit HämatoxylinEosin- oder Eisenhämatoxylin-Färbung deutlich $z \mathrm{u}$ beobachten ist, zeigt sich keineswegs das Bild der kontinuierlichen Anordnung. Bei auffallend geschrumpften Alveolen dünkt es, als ob die in Alveolenlumen bloßliegenden Blutkapillaren abgenommen seien, was aber auf den Umstand zurückgeführt wird, daß infolge der Zusammenziehung der Alveolen die betreffenden Blutkapillaren von den benachbarten Epithelzellen umgeben werden; doch tritt dabei die echte Wucherung der Epithelzellen nicht auf. Mitunter liegen die Staubzellen der Blutkapillarenwand dicht an und rufen oft das Bild hervor, als ob sie diese bedeckten, doch läßt sich auch in solchen Falle die Wucherung des Bindegewebes in der entblößten Blutkapillarenoberfläche nicht nachweisen. Nach Loosli ('38) kommen in der Alveolenwand des normalen Kaninchens die Alveolenepithelzellen in den Maschenräumen des Blutkapillarennetzes als isolierte Zellen zerstreut vor, er bestätigte auch bei der Phrenicotomie und bei dem 40 Tage lang andauernden Ṕneumothorax die diskontinuierliche Anordnung der Epithelzellen der Alveolenwand des Kaninchens. Weiter führte er an, daß der Protoplasmafortsatz dieser isolierten kernhaltigen Epithelzellen die benachbarten in Alveolenlumen bloßliegenden Blutkapillarenfläche nicht überdeckt. Dogliotti, Amprino ('32) fanden bei der Kollapslunge des Meerschweinchens und Kaninchens an der Kapillarenwand der Alveolen keine kontinuierlichen kernhaltigen Epithelzellen. Dagegen sahen Clini ('29), Bettini ('32) in der Kollapslunge des Meerschweinchens, Kaninchens und Hundes an der Blutkapillarenwand die Zellwucherung und Miller ('32) fand bei der atelektischen und pneumonischen Lunge des Menschen die kontinuierliche Auskleidung des Alveolenepithels. Low ('52), der mit Hilfe des Elektronenmikroskops die Alveolenwand der Ratte beobachtet hat, berichtet, daß das Zytoplasma des Alveolenepithels auch die Blutkapillaren der Alveolenwand überdeckt, so die Alveolenwand ein kontinuierliches Epithel besitzt. Kürzlich kam Hayek ('51) bei Meerschweinchen auf Grund der experimentellen Untersuchung des autonomen Nervensystems zu der folgenden Auffassung, daß bei der 
sauerstoffarmen Lunge die Alveolenepithelzellen kugelig werden und ein Teil der Blutkapillaren sich im Alveolenlumen entblößt, während bei hin. reichender Sauerstoffgabe die Alveolenepithelzellen Protoplasmafortsätze entsenden, die entblößten Blutkapillaren überkleiden und sich miteinander verbinden. Nach meinen Ergebnissem bei normalen Kaninchenlungen sind die Zytoplasmafortsatze der Alveolenepithelzellen oft $z \mathrm{u}$ finden, jedoch wurde das Bild, das auf die Überdeckung der Blutkapillaren durch Expansion der Zytoplasmafortsätze hindeutet, nicht gewonnen. Bei der künstlichen Pneumothoraxlunge ist es schwierig die Zytoplasmafortsätze entsendenden Epithelzellen zu finden, da dort im allgemeinen die Epithelzellen abgerundet sind. Diese Erscheinung ist beim dreimonatlichen Pneumothorax deutlicher als bei einmonatlichen, und zugleich kommen auch viele vakuolenhaltige Zellen vor. Bei der als Folge des künstlichen Pneumothorax deutlich kollabierten Lunge kommt infolge der Zusammenziehung des umgebenden Gewebes häufig das Bild zustande, das die kontinuierliche Anordnung der Alveolenepithelzellen täuscht. Ater auch in diesem Falle ist bei eingehender Beobachtung keine Wucherung der Epithelzellen auf der Blutkapillarenoberfläche $z u$ finden. Die kleinen kernhaltigen kubischen Zellen der Alveolenwand sind einzeln oder gruppenweise in den Maschenräumen des Blutkapillarennetzes vorhanden. Sind nun in der entblößten Alveolenblutkapillarenoberfläche keine Gewebselemente vorhanden? Diesbezüglich existieren viele Erörterungen. Loosli ('35) sagt, daß solche Blutkapillarenwand durch die Grundsubstanz, welche die netzartigen Bindegewebsfasern enthält, bedeckt ist. Shimai und Tamaru ('51) wiesen früher in der Menschenlunge die elastischen Fasern in der entblößten Blutkapillarenwand nach und schlossen daraus, daß das Netzwerk der Bindegewebsfasern die Blutkapillarenwand von der Alveolenlumen abtrennt. Auch bei der normalen Kaninchenlunge kamen sie zu dem gleichen Ergebnis. Loosli ('38) hat im nicht imprägnierten Präparat beobachtet, daß das Zytoplasma der Alveolenepithelien im allgemeinen hell und granuliert und in einigen Zellen auch vakuolhaltig ist. Es läßt sich jedoch auf Grund meines experimentellen Untersuchung nicht entscheiden, ob die Alveolenepithelzellen die phagozytierende Tätigkeit besitzen. Im Zytoplasma der Alveolenepithelzellen wurden neben der mit Eosin gefärbten wolkigen oder granulierten Masse keine Strukturen gefunden. Josselyn ('35) unterschied beim Alveolenepithel zwei Arten Zellen; bei der einen Art ähnelt der Kern dem der Endotholzelle der Blutkapillaren, ist etwas länger und elliptischer als der Kern der Endothelzellen von größeren Blutgefäßen und 
schwach färbbar. Die andere Art Zellen besitzt einen stark färbbaren kugeligen Kern und in dem Zytoplasma Granula. Er führte an, daß diese Zellen in der normalen Alveolenwand häufig vorkommen, den freien Zellen im Alveolenlumen ähneln, und es beinahe sicher ist, daB sie mit den sog. Septalzellen der Lunge oder den seit früher oft beschriebenen epithelialen Elementen gleichartig sind. Der Kern der Epithelzellen ist im allgemeinen chromatinreich, die Größe der Chromatingranula ist schwankend und die Verteilung im Kernraum fast gleichmäßig. Dagegen ist bei der Pneumothoraxlunge der Kern im allgemeinen heller, die Kernkörperchen sind vergrößert und beim dreimonatlichen Pneumothorax sind die Chromatingranula oft im peripherischen Teil des Kerns dichter verteilt. Der bei der normalen Lunge lumenwärts des Zelleibes exzentrisch gelegene Kern ist bei der Pneumothoraxlunge im allgemeinen im zentralen Teil des Zelleibes gefunden. Kurita ('52) berichtete bei der Kaninchenlunge nach dem einmonatlichen Pneumothorax das Hellerwerden des Kerns von Alveolenepithel und die Vergrößerung des Nukleolus.

\section{Über die Bindegewebsfasern der Alveolenwand.}

Nach Ogawa ('20) ist die normale Kaninchenlunge reich an elastischen Fasern, und die vom elastischen Faserring der Alveolenmündung nach der Alveolenwand verästelnden Fasern geben weiter feine Fäserchen in die Alveolenwand ab. Orsos ('07) teilte die elastischen Fasern der normalen Alveolenwand in zwei Systeme ein, und zwar stellt das eine das respiratorische System dar, bei welchem die aus der Wandung der Bronchien entspringenden groben Fasern unabhängig von den Kapillaren sind. Das andere System ist ein interkapillares Netzwerk, welches aus feinen Fasern besteht und von der elastischen Faserschicht der großen Gefäße verästelt. Dieses interkapillare Netzwerk bildet in Begleitung der Kapillaren ein Netzwerk, welches die Kapillaren an der Alveolenwandung fixiert. Russakoff ('09), Seemann ('31) und Josselyn ('36) nehmen an, da $B$ die kollagenen Fasern eine innige topographische Beziehung mit den elastischen Fasern zeigend das bindegewebige Fasergerüst bilden. Die funktionelle Rolle der elastischen Fasern im Lungengewebe ist schon hinreichend bekannt und benötigt hier keine weitere Erklärung. Nach meiner Beobachtung ist, im allgemeinen gesprochen, die Entwicklung der Fasern im Lungengewebe außerordentlich gut, doch zeigen die elastischen Fasern der hinreichend zusammengezogenen Alveolenwände und Alveolensepta und die Fasern in dem peribronchialen Gewebe, der Arterien- und Venenwand und Pleura pulmonalis bei dem 
dreimonatlichen Pneumothorax so auffallende Entwicklung, daß es dünkt, als ob ihre Dicke zugenommen sei. Aber die Veränderung der perikapillären Fasern der Alveolenwand ist nicht so deutlich. Kawa i ('44) bemerkte bei der geschlossenen Kollapslunge das Degenerationsbild der elastischen Fasern in der Alveolenwand, Takeda ('31) die Verdickung und Ansammlung der elastischen Fasern und Kawabata ('33) berichtete die Dilatation, Verdickung und Wucherung der intraseptalen elastischen Fasern. Shinagawa ('51) beobachtete ebenfalls die Wucherung der elastischen Fasern und unabhängig von den älteren elastischen Fasern den geradlinigen Verlauf von neugebildeten feineren Fasern. Aoki, Tokugawa und Kageyama ('51) berichteten über die Befunde der elastischen Fasern in der experimentellen Kollapslunge das Folgende: „, Die elastischen Fasern der Alveolenwand der Kollapslunge zeigen deutliche Zunahme und regressive Veränderung; sie sind parallel zu der Alveolenwand so kompakt angeordnet, daß sich eine hochgradige Wucherung annehmen läßt, während sie entsprechend der Kollapsperiode der Schlingelung, Anschwellung und Erlöschung anheimfallen. Bezüglich der Gitterfasern führte Ogawa ('26) an, daß in der normalen Lunge die Gitterfasern im allgemeinen mit den elastischen Fasern parallel laufen, sich tiefer als die elastischen Fasern befinden und gegenüber den Blutkapillaren längs oder ringförmig verlaufen. Nach Russak off ('09) lassen sich die Gitterfasern ebenfalls in zwei Systeme einteilen, das eine ist das von Blutkapillaren begleitete System, das andere das von elastischen Fasern begleitete. Außerdem bemerkte er die Blutkapillaren der Alveolenwand zirkulär umschlingenden Fasern. In seiner Untersuchung über die. Kollapslunge berichtete Kawai ('44), $\mathrm{da} B$ die Verdickung und Wucherung der Gitterfasern, unabhängig von der Luftmenge der Alveolen, bei einem längeren Verlauf nach der Operation erst zu Tage kommen. Russak off beobachtete ebenfalls bei der Pneumothoraxlunge die Verdickung oder Schlingelung der Gitterfasern. Nach meiner Beobachtung ist die Vermehrung. der Gitterfasern der Alveolenwand bei dem dreimonatlichen Pneumothorax nicht auffallend. Auch zeigen die kollagenen Fasern der Alveolenwand keine deutliche Veränderung, indessen stellen sie in der verdickten Pleura eine dichte Anordnung dar. Kurusu und Matsushige ('39) beobachteten bei Kaninchen am 10. Monate nach der Bronchienunterbindung die Vermehrung der Bindegewebsfasern und Rundzellen, während Oda ('37), Takeda, Horiuchi ('36), Kawabata ('33) u.a. die Vermehrung verneinten. Nach Kanazawa ist bei der Pneumothoraxlunge des Kaninchens im allgemeinen die Vermehrung der Binde- 
gewebsfasern nicht zu beobachten, doch hat er die Umwandlung der Gitterfasern in kollagene Fasern berichtet. Nach meiner Beobachtung der dreimonatlichen künstlichen Pneumothoraxlunge zeigen die elastischen Fasern der Alveolenwand eine geringfügige Verdickung, welche aber in der Blutkapillarenwand viel unbedeutender ist. Die Vermehrung und die Degeneration der Gitter- und kollagenen Fasern sind keineswegs zu bemerken.

\section{SchluB.}

1. Bei den Kaninchen überdecken die kernhaltigen Alveolenepithelzellen diskontinuierlich die Alveolenwand; die sog. kernlose Platte ist nicht nachzuweisen.

2. Bei der normalen Kaninchenlunge ausdehnen sich die Zytoplasmafortsätze des Alveolenepithels entlang der entblößten Blutkapillarenfläche. Bei diesem Fall bleiben noch die Blutkapillaren zum Teil in Alveolenlumen bloßliegen.

3. Die in Alveolenlumen entblößte Blutkapillarenwand scheint von einem Netzwerk von elastischen, kollagenen und Gitterfasern ausgekleidet zu sein; es ist daher denkbar, daß sie mit diesen Fasern am Alveolenlumen angrenzt.

4. Bei der künstlichen Pneumothoraxlunge des Kaninchens runden sich häufig die kernhaltigen kubischen Zellen ab, dies ist besonders deutlich der Fall bei lang andauerndem Pneumothorax.

5. Bei langdauernden Pneumothorax ist das Zytoplasma vieler Alveolenepithelzellen vakuolisiert, der Kern erscheint heller, die Kernkörperchen sind vergrößert und das Chromatin ist in dem peripheren Teil des Kernes konzentriert.

6. Die in dem zusammengezogenen Alveolenlumen befindlichen Staubzellen liegen manchmal der entblößten Blutkapillarenfläche dicht an, dort findet man doch in der Blutkapillarenwand weder die Vermehrung des interstitiellen Gewebe noch die Wucherung des Auskleidungsepithels.

7. Bei dem dreimonatigen künstlichen Pneumothorax tritt eine unbedeutende Verdickung der elastischen Fasern ein, während die Wucherung der Bindegewebsfasern kaum zu beobachten ist.

Am Schlusse dieser Arbeit möchte ich Herrn Prof. Dr. T. Taniguchi und Assistent-Prof. Dr. K. Shimai für die unermüdliche Leitung und Ratschläge meinen aufrichtigsten Dank aussprechen. 


\section{Literatur.}

1) Akazaki, K. and T. Hozaka., 1944: On the lining of the epithelial cells on the alveolar wall of the human lung. Trans. Soc. Path. Jap. Vol. 33.

2) Akutsu, K., 1933: Studien über den feineren Bau der Alveole der Wirbeltiere. Nissinigaku Bd. 22. (Japanisch).

3) Aoki, T., Tokugawa, H. and K. Kageyama., 1951: Pathological studies on influences of collapse therapy on pulmonary tuberculosis. Trans. Soc. Path. Jap. Vol. 40.

4) Baba, T., 1947: Beiträge zur Kenntnis der morphologischen und histogenetischen Studien über die Lungenalveolenepithelzellen. Zwillingsanat. Heft. 8. (Japanisch).

5) Bargmann, W., 1935: Zur vergleichenden Histologie der Lungenalveole. Z. Zellforsch. u. mikr. Anat. Bd. 23.

6) Bargmann, W., 1936: Handb. d. mikr. Anat. d. Menchen. V/3. Die Lungenalveole. Berlin.

7) Bettini, D. and A. Celotti, 1933: La histologia del polmone in condizioni fisiologische, nel pneumotorace, frenicotomia, toracoplastica. Tuberculosi. Vol. 25. (Zit. nach Loosli 1935.)

8) Bensley, R.R. and Bensley, S.H., 1935: Studies of the lining of the pulmonary alveolus of normal lungs of adult animals. Anat. Rec. Vol. 64.

9) Bermer, J.L., 1935: Postnatal development of alveoli in the mammalian lung in relation to the problem of the alveolar phagocyte. Contribution to Embryology. Vol. 25. (Zit. nach Hayek.)

10) Businco. A., 1933: La struttura del polmone alla luce delle vecchie e nuove ricerche. Monit. zoll. ital. 44.

11) Clara, M., 1936: Vergleichende Histologie des Nierenglomerulus und der Lungenalveole nach Untersuchungen beim Menschen und beim Kaninchen. $Z$. f. mikr.-anat. Forsch. Bd. 40.

12) Clini, V., 1929: Ricerche sperimentali sul pneumotorace. Boll. Soc. Ital. di Biol. Sper. Vol. 4. (Zit. nach Loosli 1935.)

13) Dogliotti, C.C. and R. Amprino., 1931: Sulla structtura dell' alveole polmonare. Bull. Histol. appl. Tome. 8.

14) Eberth, C. J., 1862: Der Streit über das Epithel der Lungenbläschen. Virschow. Arch. Bd. 24 .

15) Elenz, E., 1864: Über das Lungenepithel. Würzburg naturwiss. Zeitschr. Bd. 5.

16) Fried, B., 1934: The lungs and the macrophage system. Arch. Path. Vol. 17.

17) Hayek, H.V., 1951: Über reaktive Formänderungen der Alveolarepithelzellen bei verschiedenen Sauerstoffangebot. Zeitschr. f. Anat. und Entw. -gesch. Bd. 115.

18) Hozaka, T., 1942: Beiträge zur entwicklungsgeschichtlichen Forschung der Alveolarepithelien der Lunge. Hokuetsu Igakukaishi. Bd. 57.

19) Ishizawa, M., 1929: Neueste Kenntnis über das sog. respiratorische Epithel. Jitsuchika to Rinsho Bd. 6. (Japanisch).

20) Ishiyama, F., 1944: Akuter Lungenkollaps. Z. jap. chir. Gesellsch. Bd. 39. (Japanisch).

21) Josselyn, L.E., 1935: The nature of the pulmonary alveolar lining. Anat. Rec. Vol. 62.

22) Kammel, W., 1952: Über die Capillarenendothelien und Silberliniensysteme in den Alveolarsepten der Lunge. Z. f. Anat. u. Entw.-gesch. Bd. 116. 
23) Kanazawa, T., 1941: Experimentelle Untersuchung über den künstlichen Pneumothorax. Trans. Soc. Path. Jap. Bd. 31.

24) Kawabata, A., 1933: Morphologische Untersuchung über die Atelektase durch BronchialverschluB. Tuberculosis. Bd. 16. (Japanisch).

25) Kawai, S., 1944: Pathohistologische Untersuchung über die atelektatische Lunge durch die Stammbronchusverbindung. Kyoto Igakukai Z. Bd. 58. (Japanisch).

26) Kölliker, A., 1881: Zur Kenntnis des Baues der Lunge des Menschen. Verhandl. der physik.-medizin, Gesellsch. in Würzburg. Bd. 16.

27) Kurita, S., 1952: Experimental cytological studies on the lung epithelium. Med. Jour. Osaka Universit. Vol. 4. (Japan.)

28) Kurusu, M., Matsushige, T. und I. Iba., 1939: Über den EinfluB des einseitigen BronchialverschluB auf den Gaswechsel, das Blutgas, insbesondere die Blutstrommenge der jeder seitigen Lunge. Mitt. aus. d. med. Ak. zu Kioto. Bd. 22. (Japanisch.)

29) Lang, F. J., 1925: Über Gewebskulturen der Lunge. Arch. f. exper. Zellforsch. Bd. 2.

30) Low, F. N., 1952: Electoron microscopy of the rat lung. Anat. Rec. Vol. 113.

31) Loosli, C, G., 1935: The rabbit's lung after phrenicotomy and pneumothorax. Anat. Rec. Vol. 62.

32) Loosli, C. G., 1938: The structure of the respiratory portion of the mammalian lung with notes on the lining of the frog lung. Am. J. Anat. Vol. 62.

33) Loosli, C. G., W.E. Adames and T. M. Thornton., 1949: The histology of the dog's lung following experimental collapse. (With special reference to the nature of the alveolar lining). Anat. Rec. Vol. 105.

34) Macklin, C. C., 1936: Pulmonic alveolar epithelium. J. Thor. Surg. Vol. 6. (Zit. nach Low.)

35) Miller, W.S., 1932: The epithelium of the lower respiratory tract, Cowdry's Spec. Cytol. P. B. Hoeber, N. Y. 2nd ed., Vol. 1.

36) Miller, W.S., 1947: The Lung. C. C. Thom as, Springfield, 3, 2nd ed., Ch. 4, The air spaces. (Zit. nach Low.)

37) Müller, J., 1907: Zur vergleichenden Histologie der Lungen unserer Haussäugetiere. Arch. mikr. Anat. Bd. 69.

38) Munk, P., 1862: Über das Epithel der Lungenalveolen. Arch. f. path. Anat. und Physiol. und klin. Med. Bd. 24.

39) Ogawa, C., 1920: Contributions to the respiratory spaces of the vertebrate lungs. Amer. J. Anat. Vol. 27.

40) Oda, G., 1937: Experimentelle Studien über den massiven Lungenkollaps durch Bronchialverschluß. Z. jap. chir. Gesellsch. Bd. 38.

41) Osawa, G., 1911: Über die Epithelien der Respirationsorgane. Nissinigaku. Bd. 2.

42) Oppel, A., 1905: Lehrbuch der vergleichenden mikroskopischen Anatomie der Wirbeltiere. Bd. 6. Jena.

43) Orsos, F., 1907: Über das elastische Gerüst der normalen und der emphysematösen Lunge. Beitr. z. path. Anat. Bd. 41.

44) Peterson, H., 1935: Histologie und mikroskopische Anatomie. München.

45) Pollicard, A., 1926: Sur la nature du revetement des alvéoles pulmonaires des mammifèrés. Bull. Hist. Appl. 3.

46) Robertson, O.H., L. T. Coggeshall and E. E. Terrel1., 1933: Experimental pneumococcus lobar pneumonia in the dog. J. Clin. Invest. Vol. 12 . (Zit. nach Loosli 1938.) 
47) Roberston, O.H., and C. G. Uhley, 1936: Changes occurring in the macrophage system of the lungs in pneumococcus lobar pneumonia. J. Clin. Invest. Vol. 15. (Zit. nach Loosli 1938.)

48) Russak off, A., 1909: Über die Gitterfasern der Lunge unter normalen und pathologischen Verhältnissen. Beitr. z. path. Anat. Bd. 45.

49) Seeman, G., 1931: Histobiologie der Lungenalveole. Jena.

50) Shimai, K., and K. Tamaru., 1951: A contribution to the histological study of the alveoli of the human lung. Okajimas Fol. anat. jap. Bd. 33 .

51) Shinagawa, T., 1951 : Changes of elastic fibers in alveoli of chronic collapsed lung. Trans. Soc. Path. Japan. Bd. 40. (Japanese)

52) Takeda, R., 1931: Vergleichende Untersuchungen über die histologischen Bilder der Lunge in den verschiedenen Bedingungen. Kaibogaku Z. Bd. 3. (Japanisch)

53) Takeda, Y. und K. Horiguchi., 1936: Histologische Untersuchung über die Lunge durch die extrapleurale Stammbronchialunterbindung. Osaka Igakukai Z. Bd. 35. (Japanisch).

\section{Erklarung der Figuren.}

Fig. 1-8. Normale Kaninchenlunge.

Fig. 1. Hämatoxylin-Eosinfärbung.

Fig. 2. Hämatoxylin-Eosinfärbung. Die Epithelzellen liegen einzeln oder gruppiert. Blutkapillarenoberfläche im Alveolenlumen bloßliegend.

Fig. 3. van Geis on'sche Färbung. Netzwerk der kollagenen Fasern in der Alveolen. wand.

Fig. 4. Weigert'sche Elastinfärbung. Leichte Windung der elastischen Fasern. Peribronchiale Fasern relativ fein.

Fig. 5. Weigert'sche Elastinfärbung.

Fig. 6. van Gieson'sche Färbung.

Fig. 7. Bielschow sky-Maresch'sche Methode. Gitterfasern umgeben die entblößte Blutkapillarenoberfläche.

Fig. 9-18. Pneumothoraxlunge.

Fig. 9. Behandlungsdauer 1 Monat. Hämatoxylin-Eosinfärbung.

Fig. 10. Behandlungsdauer 1 Monat. Hyperämisch. Kern der Epithelzellen hell. Chromatin am Rande des Kerns konzentriert.

Fig. 11. Behandlungsdauer 3 Monate. Hämatoxylin-Eosinfärbung.

Abkugelung der Epithelzellen. Wände der entblöBten Blutkapillaren dünn. Staubzellen liegen nahe der Alveolenwand.

Fig. 12. Behandlungsdauer 3 Monate. van Gieson'sche Färbung.

Schleimepithelzellen der Bronchien leicht unregelmäBig angeordnet. Vermehrung der kollagenen Fasern im peribronchialer Gewebe nicht deutlich.

Fig. 13. Behandlungsdauer 1 Monat. Abkugelung der Epithelzellen schwach. Vermehrung der Bindegewebsfasern nicht deutlich.

Fig. 14. Behandlungsdauer 1 Monat. Weigert'sche Elastinfärbung. Wellenförmige, grobe, elastische Fasern und dicke Fasern reichlich vorhanden.

Fig. 15. Behandlungsdauer 1 Monat. Weigert'sche Elastinfärbung. Perivasculäre elastische Fasern etwas dick und wellig.

Fig. 16. Bielschowsky-Meresch'sche Methode.

Fig. 17. Behandlungsd auer 3 Monate. Weigert'sche Elastinfärbung. Verdickte elastische Faser. Wellenförmige Fasern der Kapillarenwand deutlich. 
Fig. 18. Behandlungsdauer 3 Monate. Bielschowsky-Maresch'sche Methode. Netzwerk der Gitterfasern der Alveolenwand deutlich.

Fig. 19-24. Kontrollunge der entgegengesetzten Seite beim Pneumothorax.

Fig. 19. Beim dreimonatigen Pneumothorax. Hämatoxylin-Eosinfärbung. Ausgedehnte Alveolenwand. Schwache Kapillarenschlingelung.

Fig. 20. Beim einmonatigen Pneumothorax. Azanfärbung. Fasern der Blutkapillarenwand deutlich ausgeprägt.

Fig. 21. Beim dreimonatigen Pneumothorax. Weigert'sche Elastinfärbung.

Fig. 22. Beim dreimonatigen Pneumothorax. Weigert'sche Elastinfärbung. Elastische Fasern der ausgedehnten Alveolenwand verlaufen ringförmig. In diesem Teil erscheinen die Fasern deutlich.

Fig. 23. Beim einmonatigen Pneumothorax. Bielschowsky-Meresch'sche Methode.

Fig. 24. Wie oben. 
Tafel I
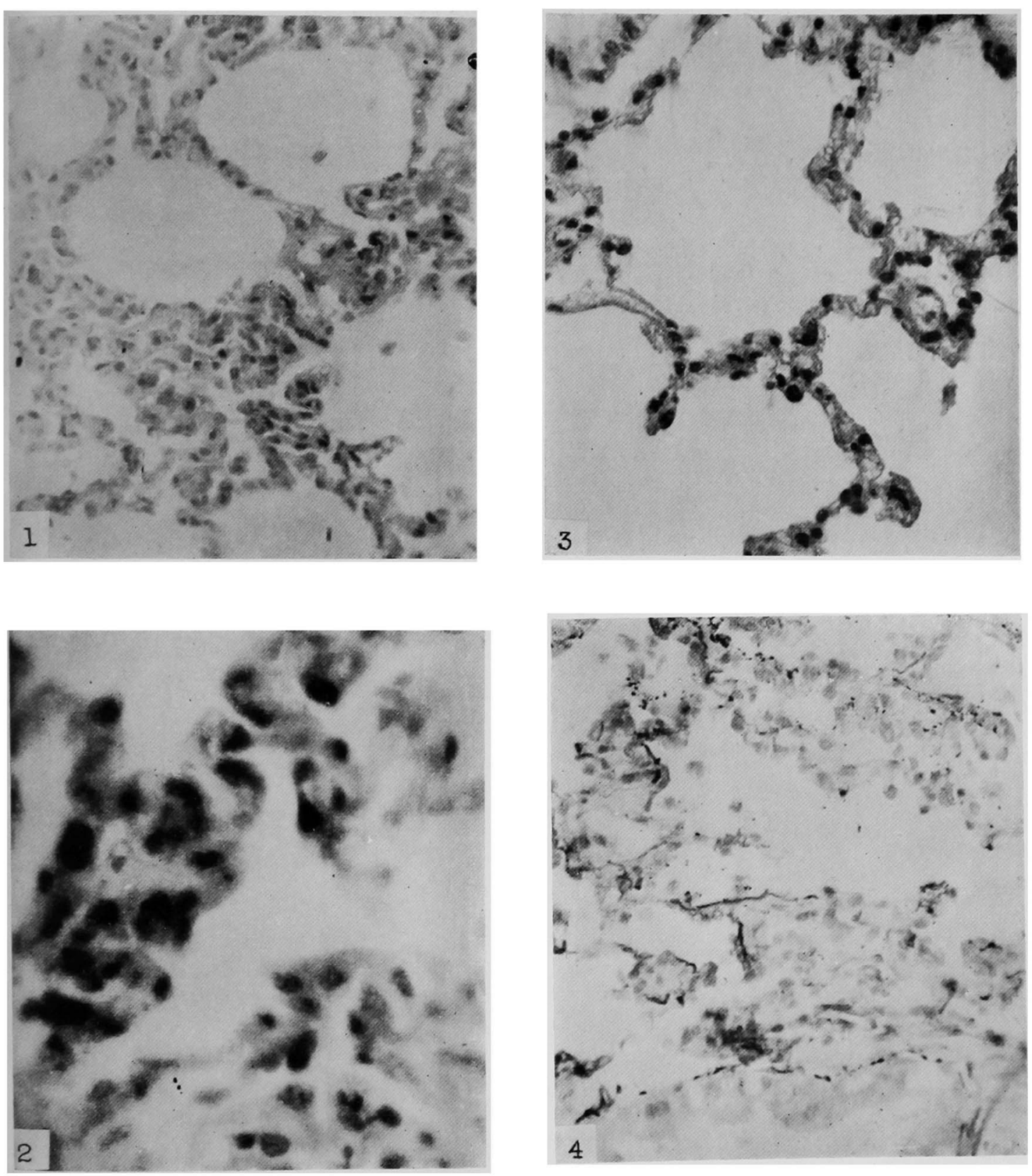

K. Tamaru 
Tafel II
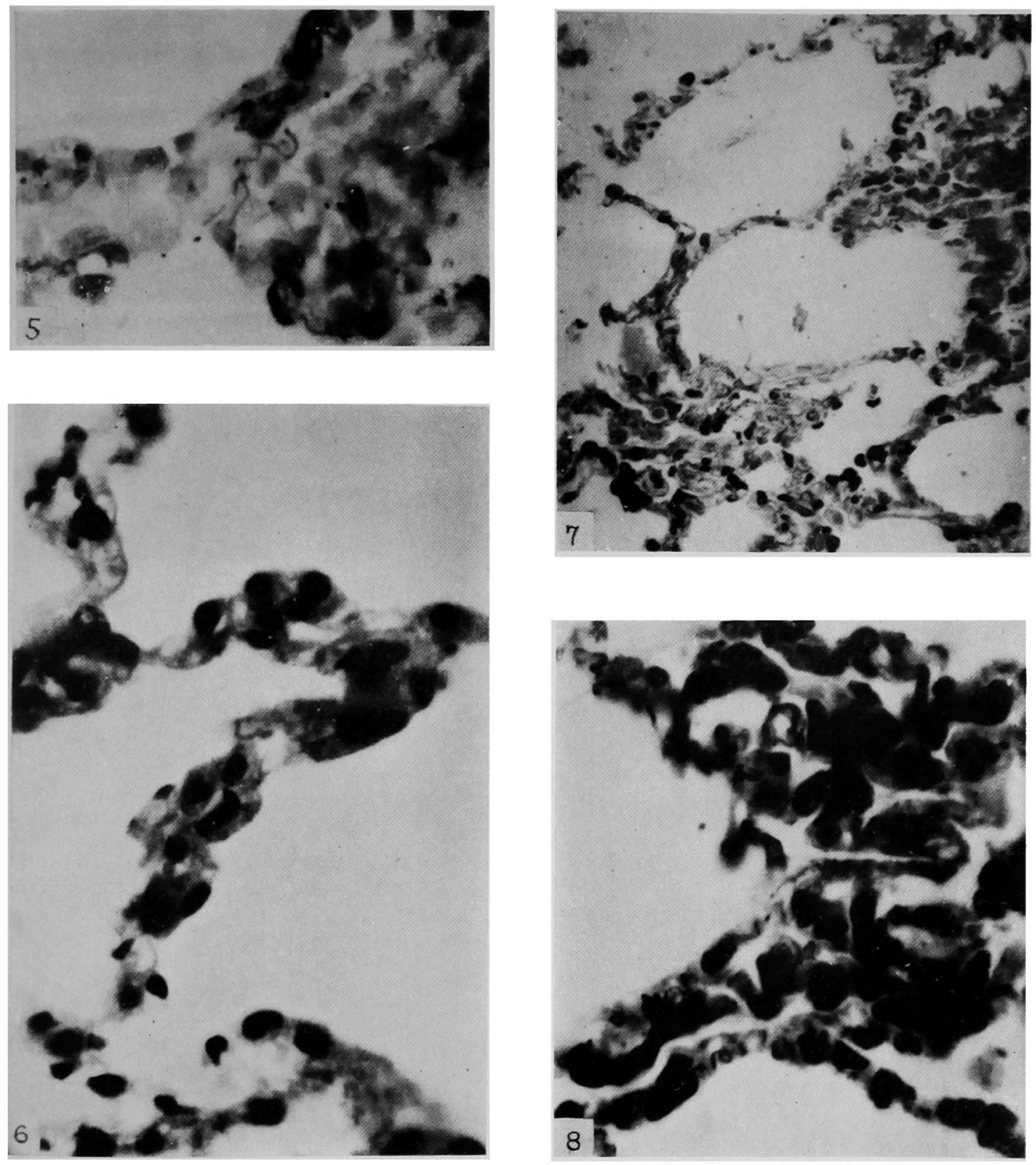

K. Tamaru 
Tafel III
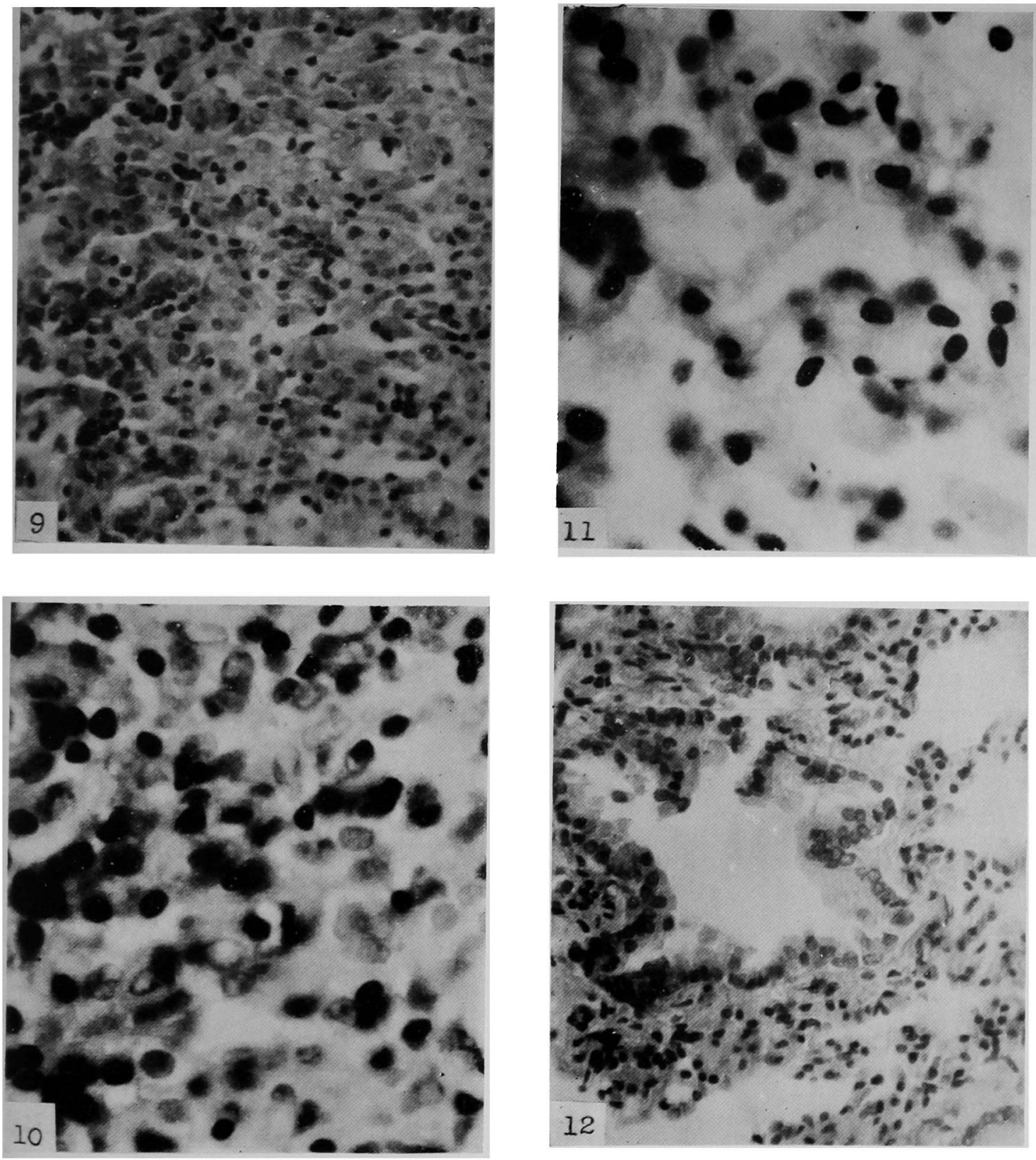

K. Tamaru 
Tafel IV
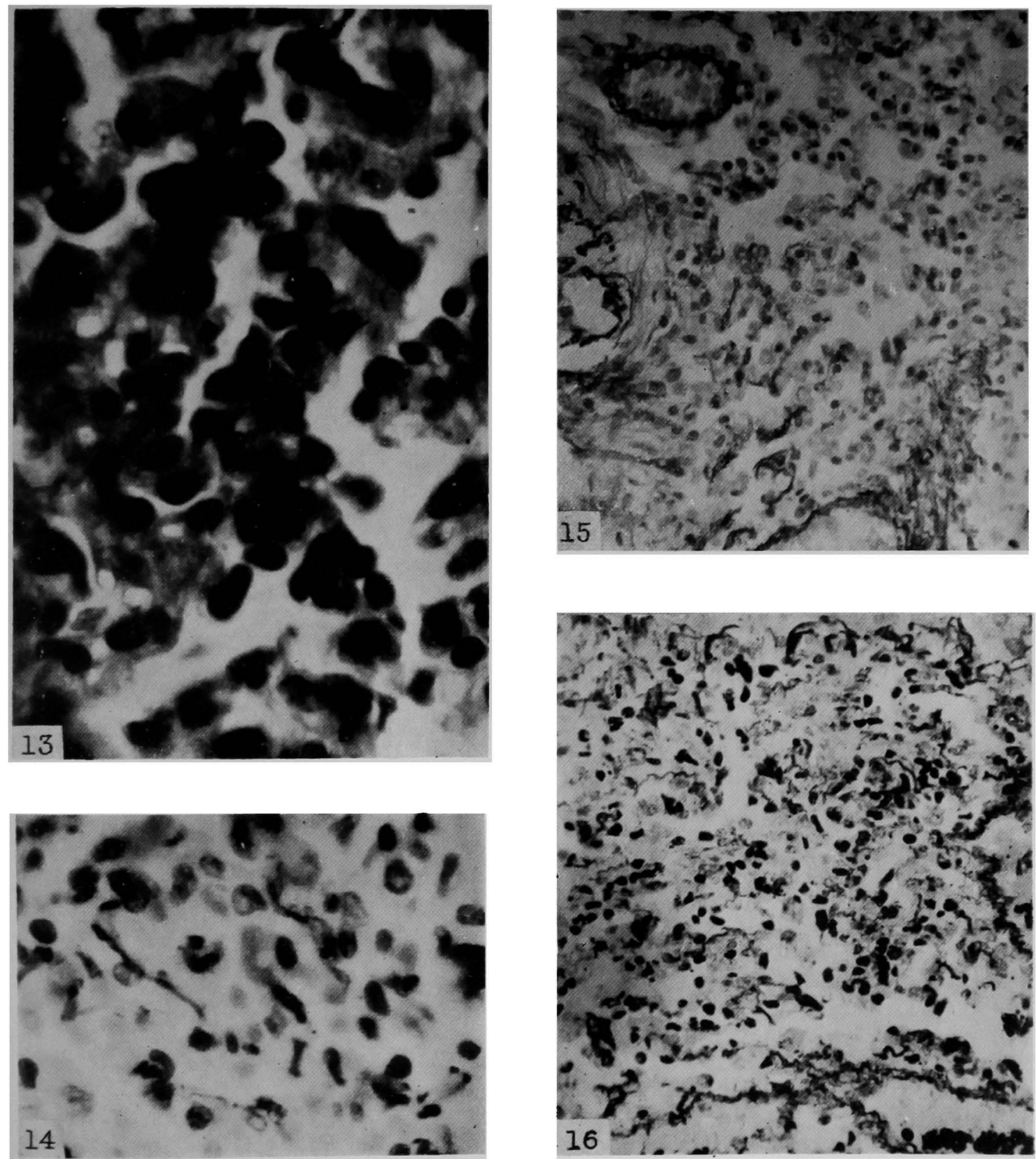

K. Tamaru 
Tafel V
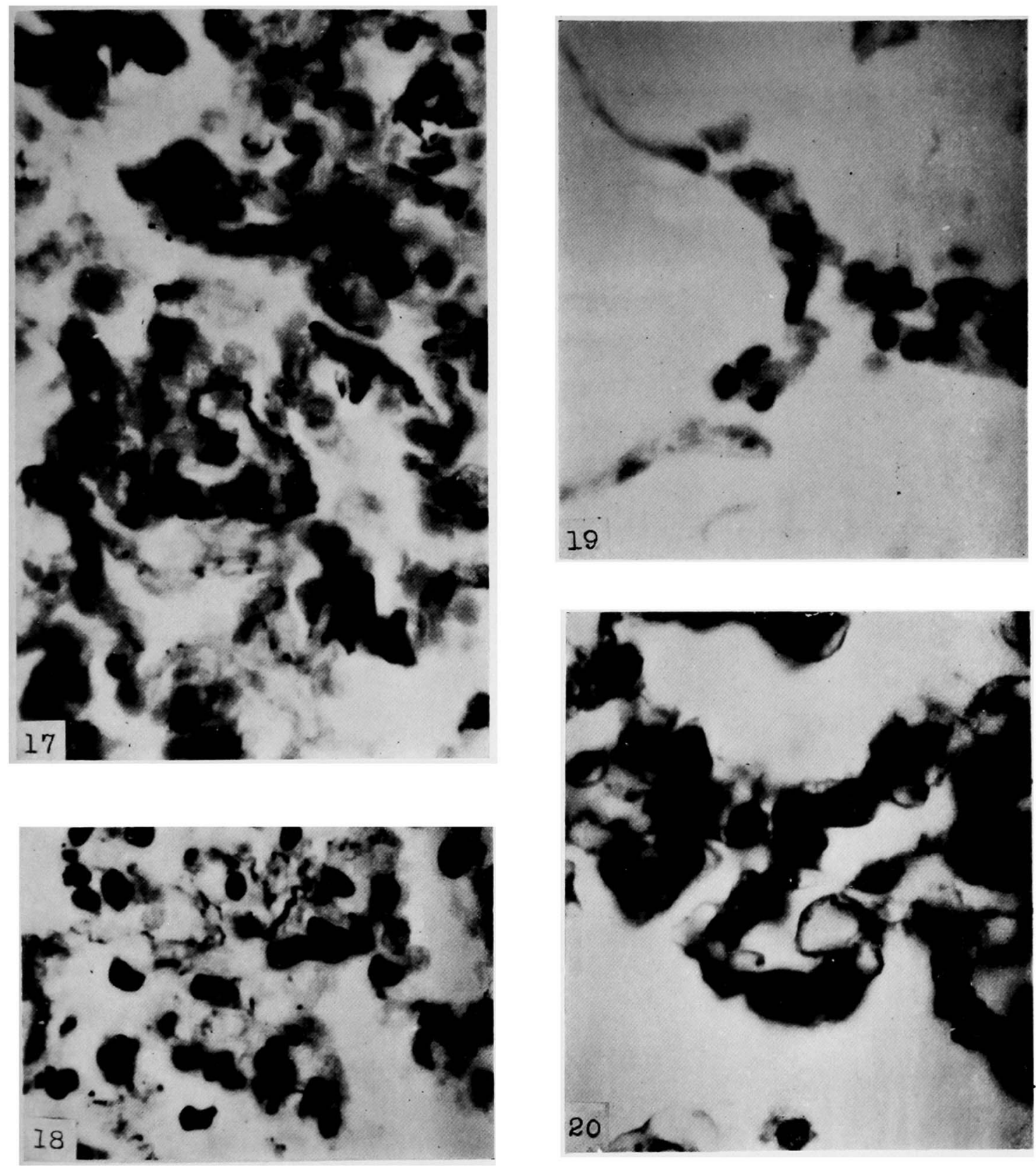

K. Tamaru 
Tafel VI
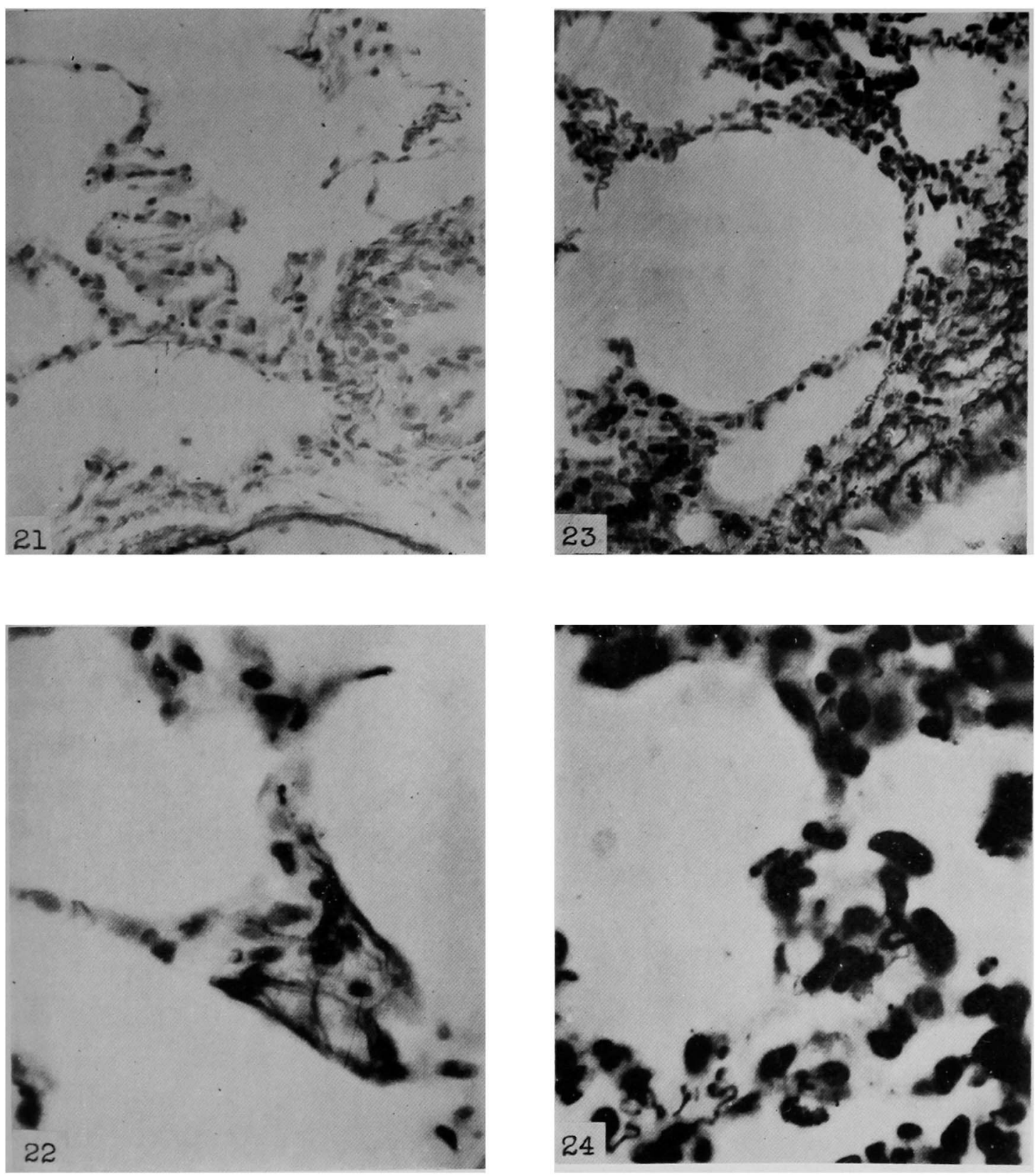

K. Tamaru 